\title{
SOME POWER SERIES INVOLVING INVOLUTIONS IN COXETER GROUPS
}

\author{
G. LUSZTIG
}

Abstract. Let $W$ be a Coxeter group. We show that a certain power series involving a sum over all involutions in $W$ can be expressed in terms of the Poincaré series of $W$. (The case where $W$ is finite has been known earlier.)

\section{INTRODUCTION}

0.1. Let $V^{\prime}$ be an $n$-dimensional vector space over the finite field $\mathbf{F}_{q}$ and let $V=$ $\mathbf{F}_{q^{2}} \otimes \mathbf{F}_{q} V^{\prime}$, an $n$-dimensional vector space over $\mathbf{F}_{q^{2}}$. Let $F: V \rightarrow V$ be the $F_{q^{-}}$-linear isomorphism $\lambda \otimes v \mapsto \lambda^{q} \otimes v$ where $\lambda \in \mathbf{F}_{q^{2}}, v \in V^{\prime}$. Let $\mathcal{F}$ be the set of all flags $V_{*}=\left(V_{1} \subset V_{2} \subset V_{n}\right)$ in $V$ where for $i=1, \ldots, n, V_{i}$ there exists an $i$-dimensional $\mathbf{F}_{q^{2}}$-subspace of $V$; let $\mathcal{F}^{\prime}$ be the analogous set defined in terms of $V^{\prime}, \mathbf{F}_{q}$ instead of $V, \mathbf{F}_{q^{2}}$. Note that $F$ induces a bijection $\mathcal{F} \rightarrow \mathcal{F}$,

$$
V_{*}=\left(V_{1} \subset V_{2} \subset V_{n}\right) \mapsto F\left(V_{*}\right)=\left(F\left(V_{1}\right) F\left(V_{2}\right) F\left(V_{n}\right)\right) .
$$

The standard way to count the number $|\mathcal{F}|$ elements in $\mathcal{F}$ is to fix $V_{*}^{0} \in \mathcal{F}$ and to partition $\mathcal{F}$ into pieces indexed by the symmetric group $S_{n}$; the piece corresponding to $w \in S_{n}$ is the set of all $V_{*} \in \mathcal{F}$ such that $V_{*}^{0}, V_{*}$ are in relative position (this piece contains exactly $q^{2 l(w)}$ elements where $l: S_{n} \rightarrow \mathbf{N}$ is the standard length function). This yields the expression $|\mathcal{F}|=\sum_{w \in S_{n}} q^{2 l(w)}$. Similarly we have $\left|\mathcal{F}^{\prime}\right|=$ $\sum_{w \in S_{n}} q^{l(w)}$.

Another way to compute $|\mathcal{F}|$ without a choice of $V_{*}^{0}$ is to partition $\mathcal{F}$ into pieces indexed by the set $I$ of involutions in $S_{n}$; the piece corresponding to $z \in I$ is the set of all $V_{*} \in \mathcal{F}$ such that $V_{*}, F\left(V_{*}\right)$ are in relative position $z$ (this piece contains exactly $\left|\mathcal{F}^{\prime}\right| q^{l(z)}\left(\frac{q-1}{q+1}\right)^{\phi(z)}$ where $\phi(z)$ is the number of $(-1)$-eigenvalues of $z$ in the reflection representation of $S_{n}$ ). (If we had taken a $z$ in $S_{n}-I$ we would have got an empty piece.) This yields the expression $|\mathcal{F}|=\left|\mathcal{F}^{\prime}\right| \sum_{z \in I} q^{l(z)}\left(\frac{q-1}{q+1}\right)^{\phi(z)}$. We deduce that the sum

$$
\sum_{z \in I} q^{l(z)}\left(\frac{q-1}{q+1}\right)^{\phi(z)}
$$

(a kind of weighted Poincaré polynomial based on involutions) is equal to a quotient of two ordinary Poincaré polynomials:

$$
\sum_{w \in S_{n}} q^{2 l(w)} / \sum_{w \in S_{n}} q^{l(w)} .
$$

The purpose of this paper is to generalize this equality to the case of Coxeter groups.

Received by the editors June 15, 2015 and, in revised form, October 17, 2015.

2010 Mathematics Subject Classification. Primary 20 G99.

Supported in part by National Science Foundation grant DMS-1303060 and by a Simons Fellowship. 
0.2. Let $(W, S)$ be a Coxeter group such that the canonical set of generators $S$ of $W$ is finite and let $w \mapsto w^{*}$ be an involutive automorphism of $W$ preserving $S$. Let $l: W \rightarrow \mathbf{N}$ be the usual length function and let $\leq$ be the standard partial order on $W$. The Poincaré series of $W$ is the formal power series $\mathbf{P}(u)=\sum_{w \in W} u^{l(w)} \in \mathbf{Z}[[u]]$ where $u$ is an indeterminate. We set $\mathbf{P}_{*}(u)=\sum_{w \in W ; w=w^{*}} u^{l(w)} \in \mathbf{Z}[[u]]$. Let $\mathbf{I}_{*}=\left\{w \in W ; w^{*}=w^{-1}\right\}$. It is easy to show (see for example [L1]) that there is a unique function $\phi: \mathbf{I}_{*} \rightarrow \mathbf{N}$ such that $\phi(1)=0$ and such that for any $w \in \mathbf{I}_{*}$ and any $s \in S$ with $s w<w$ we have $\phi(w)=\phi(s w)+1$ (if $\left.s w=w s^{*}\right)$ and $\phi(w)=\phi\left(s w s^{*}\right)$ (if $s w \neq w s^{*}$ ). We set

$$
\mathcal{R}(u)=\sum_{z \in \mathbf{I}_{*}} u^{l(z)}\left(\frac{u-1}{u+1}\right)^{\phi(z)} \in \mathbf{Z}[[u]] .
$$

(The definition of $\mathcal{R}(u)$ appeared in [L1] in the case where $W$ is finite, but the definition clearly makes sense in the general case.) We say that $W$, * has property $\mathbf{X}$ if

$$
\mathcal{R}(u)=\mathbf{P}\left(u^{2}\right) / \mathbf{P}_{*}(u) .
$$

In [L1] it was proved (case by case) that $W, *$ has property $\mathbf{X}$ if $W$ is finite. In [MW] it is proved that $W, 1$ has property $\mathbf{X}$ if $W$ is an affine Weyl group of type $A$ and the question of the validity of $\mathbf{X}$ for any $W, *$ is raised. We state the following result.

Theorem 0.3. $W, *$ has property $\mathbf{X}$.

The proof is given in 1.11. It is based on results in [LV], [L1]. The most complicated part of the proof is a property stated in Proposition 1.8. In Section 2 we give an alternative proof of this property assuming that $W$ is a Weyl group. (The same proof applies in the case where $W$ is the "Weyl group" associated to a Kac-Moody Lie algebra and $*$ is induced by an involutive automorphism of that Lie algebra.)

\section{The polynomials $X_{y}^{z}$}

1.1. Let $W, S, *, l, \mathbf{I}_{*}$ be as in 0.2 . Let $u$ be an indeterminate.

Let $\mathcal{A}=\mathbf{Z}\left[u, u^{-1}\right]$. Let $\mathfrak{H}$ be the free $\mathcal{A}$-module with basis $\left(T_{w}\right)_{w \in W}$ with the unique $\mathcal{A}$-algebra structure with unit $T_{1}$ such that

$$
\begin{gathered}
T_{w} T_{w^{\prime}}=T_{w w^{\prime}} \text { if } l\left(w w^{\prime}\right)=l(w)+l\left(w^{\prime}\right) \text { and } \\
\left(T_{s}+1\right)\left(T_{s}-u^{2}\right)=0 \text { for all } s \in S .
\end{gathered}
$$

(This is an Iwahori-Hecke algebra.) Let $M$ be the free $\mathcal{A}$-module with basis $\left\{a_{w} ; w \in\right.$ $\left.\mathbf{I}_{*}\right\}$. According to [LV] (when $W$ is a Weyl group) and [L1] (in the general case) there is a unique $\mathfrak{H}$-module structure on $M$ (extending the $\mathcal{A}$-module structure) such that for any $s \in S$ and any $z \in \mathbf{I}_{*}$ we have

(i) $T_{s} a_{z}=u a_{z}+(u+1) a_{s z}$ if $s z=z s^{*}>z$;

(ii) $T_{s} a_{z}=\left(u^{2}-u-1\right) a_{z}+\left(u^{2}-u\right) a_{s z}$ if $s z=z s^{*}<z$;

(iii) $T_{s} a_{z}=a_{s z s^{*}}$ if $s z \neq z s^{*}>z$;

(iv) $T_{s} a_{z}=\left(u^{2}-1\right) a_{z}+u^{2} a_{s z s^{*}}$ if $s z \neq z s^{*}<z$.

For any $m \in M$ we write $m=\sum_{z \in \mathbf{I}_{*}}\left(a_{z}: m\right) a_{z}$ where $\left(a_{z}: m\right) \in \mathcal{A}$ are zero for all but finitely many $z$.

Proposition 1.2. There is a unique collection of polynomials $X_{y}^{z} \in \mathbf{Z}[u]$ indexed by $(z, y) \in \mathbf{I}_{*} \times W$ such that 
( $X_{1}^{z}=\delta_{z, 1}$ for all $z \in \mathbf{I}_{*}$

and such that for any $s \in S, y \in W$ with $y>$ ys and any $z \in \mathbf{I}_{*}$ we have

(i) $X_{y}^{z}=u X_{y s}^{z}+(u+1) X_{y s}^{s z}$ if $s z=z s^{*}>z$;

(ii) $X_{y}^{z}=\left(u^{2}-u-1\right) X_{y s}^{z}+\left(u^{2}-u\right) X_{y s}^{s z}$ if $s z=z s^{*}<z$;

(iii) $X_{y}^{z}=X_{y s}^{s z s^{*}}$ if $s z \neq z s^{*}>z$;

(iv) $X_{y}^{z}=\left(u^{2}-1\right) X_{y s}^{z}+u^{2} X_{y s}^{s z s^{*}}$ if $s z \neq z s^{*}<z$.

Moreover, we have $X_{y}^{z} \in u^{l(z)} \mathbf{Z}[u]$ for any $(z, y) \in \mathbf{I}_{*} \times W$.

For $(z, y) \in \mathbf{I}_{*} \times W$ we set $X_{y}^{z}=\left(a_{1}: T_{y} a_{z}\right) \in \mathcal{A}$. Then clearly $(\star)$ holds. If $s \in S, y \in W$ satisfy $y>y s$, then $T_{y}=T_{y s} T_{s}$; hence applying $T_{y s}$ to the formulas 1.1(i)-(iv) we see that $X_{y}^{z}$ satisfy (i)-(iv) (but they are in $\mathcal{A}$ instead of $\mathbf{Z}[u]$ ). Now from $(\star)$ and (i)-(iv) we see by induction on $l(y)$ that $X_{y}^{z}$ are uniquely determined and that $X_{y}^{z} \in u^{l(z)} \mathbf{Z}[u]$ for any $(z, y) \in \mathbf{I}_{*} \times W$. The proposition is proved.

1.3. The $\mathcal{A}$-module $M$ in 1.1 can be viewed as an $\mathcal{A}$-submodule of $\hat{M}$ which consists of all formal $\mathcal{A}$-linear combinations of the elements $\left\{a_{z} ; z \in \mathbf{I}_{*}\right\}$. The $\mathfrak{H}$-module structure on $M$ extends in an obvious way to an $\mathfrak{H}$-module structure on $\hat{M}$. We show that

$$
T_{y}\left(\sum_{z} a_{z}\right)=u^{2 l(y)} \sum_{z} a_{z} \text { for any } y \in W .
$$

It is enough to prove this in the case where $y=s \in S$. For any $z \in \mathbf{I}_{*}$ we set $s \bullet z=s z$ if $s z=z s^{*}$ and $s \bullet z=s z s^{*}$ if $s z \neq z s^{*}$. From the definition for any $z \in \mathbf{I}_{*}$ such that $s z<z$ we have

$$
T_{s}\left(a_{z}+a_{s \bullet z}\right)=u^{2}\left(a_{z}+a_{s \bullet z}\right) ;
$$

hence

$$
T_{s}\left(\sum_{z \in \mathbf{I}_{*}} a_{z}\right)=\sum_{z \in \mathbf{I}_{*} ; s z<z} T_{s}\left(a_{z}+a_{s \bullet z}\right)=\sum_{z \in \mathbf{I}_{*} ; s z<z} u^{2}\left(a_{z}+a_{s \bullet z}\right)=u^{2} \sum_{z \in \mathbf{I}_{*}} a_{z} .
$$

This completes the proof of (a).

For $\hat{m} \in \hat{M}$ we write $\hat{m}=\sum_{z \in \mathbf{I}_{*}}\left(a_{z}: \hat{m}\right) a_{z}$ where $\left(a_{z}: \hat{m}\right) \in \mathcal{A}$. Applying $\left(a_{1}: ?\right)$ to (a) we obtain for any $y \in W$ :

$$
\sum_{z \in \mathbf{I}_{*}} X_{y}^{z}=u^{2 l(y)} .
$$

Note that in the left-hand side we have $X_{y}^{z}=0$ for all but finitely many $z$. Indeed, if $(z, y) \in \mathbf{I}_{*} \times W$ and $l(z)>2 l(y)$, then $X_{y}^{z}=0$. (This can be seen from Proposition 1.7 or directly from definitions by induction on $l(y)$.)

Proposition 1.4. Let $s \in S, z \in \mathbf{I}_{*}$ be such that $s z<z$ and let $y \in W$. We have:

(i) $(u+1) X_{y}^{z}=-u X_{y}^{s z}+X_{y s}^{s z}$ if $s z=z s^{*}$,ys $>y$;

(ii) $(u+1) X_{y}^{z}=u^{2} X_{y s}^{s z}+\left(u^{2}-u-1\right) X_{y}^{s z}$ if $s z=z s^{*}$,ys $<y$;

(iii) $X_{y}^{z}=X_{y s}^{s z s^{*}}$ if $s z \neq z s^{*}, y s>y$;

(iv) $X_{y}^{z}=u^{2} X_{y s}^{s z s^{*}}+\left(u^{2}-1\right) X_{y}^{s z s^{*}}$ if $s z \neq z s^{*}, y s<y$.

From 1.2(i) we obtain $X_{y s}^{s z}=u X_{y}^{s z}+(u+1) X_{y}^{z}$ if $s z=z s^{*}, y s>y$. Clearly this yields (i). In 1.2(ii) we substitute $X_{y s}^{z}=-\frac{u}{u+1} X_{y s}^{s z}+\frac{1}{u+1} X_{y}^{s z} \in \mathbf{Q}(u)$ (with $y s<y$ ) 
which follows from (i); we obtain

$$
\begin{aligned}
X_{y}^{z} & =\left(u^{2}-u-1\right)\left(-\frac{u}{u+1} X_{s y}^{s z}+\frac{1}{u+1} X_{y}^{s z}\right)+\left(u^{2}-u\right) X_{y s}^{s z} \\
& =\frac{u^{2}}{u+1} X_{y s}^{s z}+\frac{u^{2}-u-1}{u+1} X_{y}^{s z}
\end{aligned}
$$

so that (ii) holds. Now (iii) clearly follows from 1.2(iii). In 1.2(iv) we substitute $X_{y s}^{z}=X_{y}^{s z s^{*}}$ which follows from (iii); we obtain (iv). The proposition is proved.

From the proposition we deduce the following result.

Corollary 1.5. Let $z \in \mathbf{I}_{*}, y \in W, s \in S$. We have:

(i) $X_{y}^{z}+X_{y s}^{z}=u^{2}\left(X_{y}^{s z s^{*}}+X_{y s}^{s z s^{*}}\right)$ if $s z \neq z s^{*}<z$;

(ii) $(u+1)\left(X_{y}^{z}+X_{y s}^{z}\right)=\left(u^{2}-u\right)\left(X_{y}^{s z}+X_{y s}^{s z}\right)$ if $s z=z s^{*}<z$.

1.6. For any $h \in \mathfrak{H}$ we write $h=\sum_{w \in W}\left[T_{w}: h\right] T_{w}$ where $\left[T_{w}: h\right] \in \mathcal{A}$ are zero for all but finitely many $w$.

For $z^{\prime} \in \mathbf{I}_{*}, s \in S$ we show:

(i) $T_{s} T_{z^{\prime}} T_{s^{*}}=c T_{z^{\prime}}+c^{\prime} T_{s z^{\prime}}$ where $c, c^{\prime} \in \mathbf{Z}[u]-\{0\}$, if $s z^{\prime}=z^{\prime} s^{*}$;

(ii) $T_{s} T_{z^{\prime}} T_{s^{*}}=T_{s z^{\prime} s^{*}}$ if $s z^{\prime} \neq z^{\prime} s^{*}>z$;

(iii) $T_{s} T_{z^{\prime}} T_{s^{*}}=u^{4} T_{s z^{\prime} s^{*}}+\left(u^{2}-1\right)^{2} T_{z^{\prime}}+u^{2}\left(u^{2}-1\right) T_{s z^{\prime}}+u^{2}\left(u^{2}-1\right) T_{z^{\prime} s^{*}}$ if $s z^{\prime} \neq z^{\prime} s^{*}<z^{\prime}$.

In (i) assume first that $s z^{\prime}>z^{\prime}$. Then $T_{s} T_{z^{\prime}} T_{s^{*}}=T_{s} T_{z^{\prime} s^{*}}=u^{2} T_{z^{\prime}}+\left(u^{2}-1\right) T_{s z^{\prime}}$. Now assume that in (i) we have $s z<z$. Then

$$
\begin{aligned}
T_{s} T_{z^{\prime}} T_{s^{*}} & =T_{s} T_{s} T_{s z^{\prime}} T_{s^{*}}=T_{s} T_{s} T_{z^{\prime}}=u^{2} T_{z^{\prime}}+\left(u^{2}-1\right) T_{s} T_{z^{\prime}} \\
& =u^{2} T_{z^{\prime}}+u^{2}\left(u^{2}-1\right) T_{s z^{\prime}}+\left(u^{2}-1\right)^{2} T_{z^{\prime}} .
\end{aligned}
$$

Now (ii) is obvious. In (iii) we have

$$
\begin{aligned}
T_{s} T_{z^{\prime}} T_{s^{*}} & =T_{s} T_{s} T_{s z^{\prime} s^{*}} T_{s^{*}} T_{s^{*}} \\
& =u^{4} T_{s z^{\prime} s^{*}}+\left(u^{2}-1\right)^{2} T_{z^{\prime}}+u^{2}\left(u^{2}-1\right) T_{s z^{\prime}}+u^{2}\left(u^{2}-1\right) T_{z^{\prime} s^{*}},
\end{aligned}
$$

as desired.

For $s \in S$ and $z, z^{\prime} \in \mathbf{I}_{*}$ we show:

(a) If $\left(a_{z}: T_{s} a_{z^{\prime}}\right) \neq 0$, then $\left[T_{z}: T_{s} T_{z^{\prime}} T_{s^{*}}\right] \neq 0$.

If $s^{\prime} z=z^{\prime} s^{*}$, then by Proposition 1.2 we have $z=z^{\prime}$ or $z=s z^{\prime}$ and (a) follows from (i).

If $s z^{\prime} \neq z^{\prime} s^{*}>z^{\prime}$, then by Proposition 1.2 we have $z=s z^{\prime} s^{*}$ and (a) follows from (ii).

If $s z^{\prime} \neq z^{\prime} s^{*}<z^{\prime}$, then by Proposition 1.2 we have $z=z^{\prime}$ or $z=s z^{\prime} s^{*}$ and (a) follows from (iii).

This proves (a).

We shall also need a variant of (a). Assume that $s \neq s^{\prime}$ in $S$ are such that $s^{\prime}=s^{*}$, $s, s^{\prime}$ generate a finite (dihedral) subgroup of $W$ and $\sigma$ is the longest element in that subgroup. Then:

(b) If $z, z^{\prime} \in \mathbf{I}_{*}$ and $\left(a_{z}: T_{\sigma} a_{z^{\prime}}\right) \neq 0$, then $\left[T_{z}: T_{\sigma} T_{z^{\prime}} T_{\sigma^{*}}\right] \neq 0$.

(c) $\left(a_{1}: T_{\sigma} a_{1}\right)=u^{l(\sigma)}$.

This can be proved using the results in [L1] which describe the action of $T_{\sigma}$ on $M$.

We have the following result.

Proposition 1.7. If $z \in \mathbf{I}_{*}, y \in W$ and $\left(a_{z}: T_{y} a_{1}\right) \neq 0$, then $\left[T_{z}: T_{y} T_{y^{*-1}}\right] \neq 0$. 
We argue by induction on $l(y)$. If $y=1$ the result is obvious. Assume now that $y \neq 1$. We can find $s \in S$ such that $s y<y$. We have

$$
\left(a_{z}: T_{y} a_{1}\right)=\sum_{z^{\prime} \in \mathbf{I}^{*}}\left(a_{z}: T_{s} a_{z^{\prime}}\right)\left(a_{z^{\prime}}: T_{s y} a_{1}\right) .
$$

By assumption there exists $z^{\prime} \in \mathbf{I}^{*}$ such that $\left(a_{z}: T_{s} a_{z^{\prime}}\right) \neq 0$ and $\left(a_{z^{\prime}}: T_{s y} a_{1}\right) \neq 0$. By 1.6(a) we then have $\left[T_{z}: T_{s} T_{z^{\prime}} T_{s^{*}}\right] \neq 0$ and by the induction hypothesis we have $\left[T_{z^{\prime}}: T_{s y} T_{y^{*-1} s^{*}}\right] \neq 0$. We have

$$
\left[T_{z}: T_{y} T_{y^{*-1}}\right]=\sum_{w \in W}\left[T_{z}: T_{s} T_{w} T_{s^{*}}\right]\left[T_{w}: T_{s y} T_{y^{*-1} s^{*}}\right] .
$$

This is a sum of terms in $\mathbf{N}[u-1]$ (the set of polynomials in $u-1$ with coefficients in $\mathbf{N}$ ) and the term corresponding to $w=z^{\prime}$ is nonzero; hence the sum is nonzero. The proposition is proved.

Proposition 1.8. For any $y \in W$ we have $X_{y}^{1}=\delta_{y, y^{*}} u^{l(y)}$.

From [L2, 10.4(a)] we have

$$
\left[T_{1}: T_{y_{1}} T_{y_{1}^{*-1}}\right]=\delta_{y_{1}, y_{1}^{*}} u^{2 l\left(y_{1}\right)} \text { for any } y_{1} \in W \text {. }
$$

We prove the proposition by induction on $l(y)$. If $y=1$ the result is obvious. Assume now that $y \neq 1$. We can write $y=\sigma y^{\prime}$ where $\sigma, y^{\prime} \in W, l(y)=l(\sigma)+l\left(y^{\prime}\right)$ and the following holds: if $y \neq y^{*}$, then $\sigma \in S$; if $y=y^{*}$, then there exists $s, s^{\prime}$ in $S$ such that $s^{\prime}=s^{*}, s, s^{\prime}$ generate a finite subgroup of $W$ and $\sigma$ is the longest element in that subgroup, so that $\sigma=\sigma^{*}$. (We use [L2, A1(a)].) By the induction hypothesis we have

$$
T_{y^{\prime}} a_{1}=\delta_{y^{\prime}, y^{\prime} *} u^{l\left(y^{\prime}\right)} a_{1}+\sum_{z \in \mathbf{I}_{*}, z \neq 1}\left(a_{z}: T_{y^{\prime}} a_{1}\right) a_{z} .
$$

Since $T_{y}=T_{\sigma} T_{y^{\prime}}$, it follows (also using (a)) that

$$
\begin{gathered}
T_{y} a_{1}=\delta_{y^{\prime}, y^{\prime *}} u^{l\left(y^{\prime}\right)} T_{\sigma} a_{1}+\sum_{z \in \mathbf{I}_{*}, z \neq 1}\left(a_{z}: T_{y^{\prime}} a_{1}\right) T_{\sigma} a_{z}, \\
T_{y} T_{y^{*-1}}=\delta_{y^{\prime}, y^{\prime *}} u^{2 l\left(y^{\prime}\right)} T_{\sigma} T_{\sigma^{*}}+\sum_{w \in W ; w \neq 1}\left[T_{w}: T_{y^{\prime}} T_{y^{\prime *-1}}\right] T_{\sigma} T_{w} T_{\sigma^{*}} .
\end{gathered}
$$

Note that by (a) we have

$$
\left[T_{1}: T_{\sigma} T_{\sigma^{*}}\right]=\delta_{\sigma, \sigma^{*}} u^{2 l(\sigma)} .
$$

Hence $\gamma:=\left[T_{1}: \delta_{y^{\prime}, y^{\prime *}} u^{2 l\left(y^{\prime}\right)} T_{\sigma} T_{\sigma^{*}}\right]$ is $\delta_{y^{\prime}, y^{*}} u^{2 l\left(y^{\prime}\right)} \delta_{\sigma, \sigma^{*}} u^{2 l(\sigma)}$, that is $\delta_{y, y^{*}} u^{2 l(y)}$. (If $y \neq y^{*}$ we have $\gamma=0$ since we have either $\delta_{y^{\prime}, y^{\prime *}}=0$ or $\delta_{\sigma, \sigma^{*}}=0$. If $y=y^{*}$, then $\sigma=\sigma^{*}$ hence $y^{\prime}=y^{* *}$ and $\gamma=u^{2 l(y)}$.)

Assume that for some $z \in \mathbf{I}_{*}-\{1\}$, we have $\left(a_{z}: T_{y^{\prime}} a_{1}\right) \neq 0$ and $\left(a_{1}: T_{\sigma} a_{z}\right) \neq 0$. Then, by Proposition 1.7 we have $\left[T_{z}: T_{y^{\prime}} T_{y^{\prime *}}\right] \neq 0$. Moreover, we have $\left[T_{1}\right.$ : $\left.T_{\sigma} T_{z} T_{\sigma^{*}}\right] \neq 0$. (When $\sigma \in S$ this follows from 1.6(a). When $\sigma \notin S$ this follows from 1.6(b).) Thus,

$$
\pi:=\left[T_{1}: \sum_{w \in W ; w \neq 1}\left[T_{w}: T_{y^{\prime}} T_{y^{\prime *-1}}\right] T_{\sigma} T_{w} T_{\sigma^{*}}\right]
$$

is a sum of terms in $\mathbf{N}[u-1]$, at least one of which is $\neq 0$, so that $\pi \in \mathbf{N}[u-1]-\{0\}$. Thus $\left[T_{1}: T_{y} T_{y^{*-1}}\right]=\delta_{y, y^{*}} u^{2 l(y)}+\pi$. By (a), $\left[T_{1}: T_{y} T_{y^{*-1}}\right]=\delta_{y, y^{*}} u^{2 l(y)}$. Thus, $\pi=0$, a contradiction. 
This contradiction shows that for any $z \in \mathbf{I}_{*}-\{1\}$ we have either $\left(a_{z}: T_{y^{\prime}} a_{1}\right)=$ 0 or $\left(a_{1}: T_{\sigma} a_{z}\right)=0$. Hence from (b) we deduce that $\left(a_{1}: T_{y} a_{1}\right)=\left(a_{1}\right.$ : $\left.\delta_{y^{\prime}, y^{\prime *}} u^{l\left(y^{\prime}\right)} T_{\sigma} a_{1}\right)$. We have

$$
\left(a_{1}: T_{\sigma} a_{1}\right)=\delta_{\sigma, \sigma^{*}} u^{l(\sigma)} .
$$

(When $\sigma \in S$ this follows from 1.1(i)-(iv). When $\sigma \notin S$ this follows from 1.6(c).) We deduce

$$
\left(a_{1}: T_{y} a_{1}\right)=\delta_{y^{\prime}, y^{\prime} *} u^{l\left(y^{\prime}\right)} \delta_{\sigma, \sigma^{*}} u^{l(\sigma)}=\delta_{y^{\prime}, y^{\prime *}} \delta_{\sigma, \sigma^{*}} u^{l(y)} .
$$

It remains to show that

$$
\delta_{y^{\prime}, y^{\prime *}} \delta_{\sigma, \sigma^{*}}=\delta_{y, y^{*}}
$$

If $y \neq y^{*}$, then we have either $y^{\prime} \neq y^{* *}$ or $\sigma \neq \sigma^{*}$; hence both sides of (c) are zero. If $y=y^{*}$, then $\sigma=\sigma^{*}$ hence $y^{\prime}=y^{\prime *}$ hence both sides of (c) are 1. Thus (c) holds. The proposition is proved.

Proposition 1.9. We have $X_{y}^{z} \in u^{l(y)} \mathbf{Z}[u]$ for any $(z, y) \in \mathbf{I}_{*} \times W$.

This follows by induction on $l(z)$ using 1.4(i)-(iv); to start the induction, we assume that $z=1$ in which case the result follows from Proposition 1.8.

1.10. For any $z \in \mathbf{I}_{*}$ we set

$$
X^{z}=\sum_{y \in W} X_{y}^{z} \in \mathbf{Z}[[u]]
$$

Note that, by Proposition 1.9, the sum in the right-hand side converges in $\mathbf{Z}[[u]]$. For $z \in \mathbf{I}_{*}$ we show that

$$
X^{z}=\mathbf{P}_{*}(u) u^{l(z)}\left(\frac{u-1}{u+1}\right)^{\phi(z)} .
$$

We argue by induction on $l(z)$. If $z=1$ we have, using Proposition 1.8:

$$
X^{1}=\sum_{y \in W ; y=y^{*}} u^{l(y)}=\mathbf{P}_{*}(u) .
$$

Assume now that $z \neq 1$. We can find $s \in S$ such that $s z<z$. From Corollary 1.5 we deduce that $X^{z}=u^{2} X^{s z s^{*}}$ if $s z \neq z s^{*}$ and $(u+1) X^{z}=\left(u^{2}-u\right) X^{s z}$ if $s z=z s^{*}$. Using the induction hypothesis we see that, if $s z \neq z s^{*}$ we have

$$
X^{z}=\mathbf{P}_{*}(u) u^{l\left(s z s^{*}\right)+2}\left(\frac{u-1}{u+1}\right)^{\phi\left(s z s^{*}\right)},
$$

while if $s z=z s^{*}$ we have

$$
X^{z}=\mathbf{P}_{*}(u) u^{l(s z)+1}\left(\frac{u-1}{u+1}\right)^{\phi(s z)+1} ;
$$

the desired result follows. 
1.11. We prove Theorem 0.3. The sum $\sum_{(z, y) \in \mathbf{I}_{*} \times W} X_{y}^{z}$ is convergent in $\mathbf{Z}[[u]]$ since $X_{y}^{z} \in u^{\max (l(z), l(y))} \mathbf{Z}[u]$ (see Propositions 1.2 and 1.9). We can compute this sum in two different ways and we get the same result. Thus we have

$$
\sum_{z \in \mathbf{I}_{*}}\left(\sum_{y \in W} X_{y}^{z}\right)=\sum_{y \in W}\left(\sum_{z \in \mathbf{I}_{*}} X_{y}^{z}\right)
$$

By 1.3(b), the right-hand side is equal to $\sum_{y \in W} u^{2 l(y)}=\mathbf{P}\left(u^{2}\right)$. By 1.4(c), the left-hand side is equal to

$$
\sum_{z \in \mathbf{I}_{*}} \mathbf{P}_{*}(u) u^{l(z)}\left(\frac{u-1}{u+1}\right)^{\phi(z)}=\mathbf{P}_{*}(u) \mathcal{R}(u) .
$$

Thus we have

$$
\mathbf{P}\left(u^{2}\right)=\mathbf{P}_{*}(u) \mathcal{R}(u) .
$$

Theorem 0.3 is proved.

\section{The CASE OF Weyl Groups}

2.1. In this section we assume that $W$ is the Weyl group of a connected adjoint simple algebraic group $\mathbf{G}$ defined and split over the finite field $\mathbf{F}_{p}$ with $p$ elements ( $p$ is a prime number); we identify $\mathbf{G}$ with $\mathbf{G}(\mathbf{k})$ where $\mathbf{k}$ is an algebraic closure of $\mathbf{F}_{p}$. Let $F: \mathbf{G} \rightarrow \mathbf{G}$ be the "Frobenius map"; it is an abstract group isomorphism whose fixed point set is the group of $\mathbf{F}_{p}$-rational points of $\mathbf{G}$. Let $\mathcal{B}$ be the variety of Borel subgroups of $\mathbf{G}$. The set of $\mathbf{G}$-orbits on $\mathcal{B} \times \mathcal{B}$ (for the simultaneous conjugation action) are naturally indexed by $W$; we denote by $\mathcal{O}_{w}$ the $\mathbf{G}$-orbit indexed by $w \in W$. The length function $l: W \rightarrow \mathbf{N}$ is such that for any $w \in W$ and any $C \in \mathcal{B}$, the algebraic variety $\left\{B \in \mathcal{B} ;(C, B) \in \mathcal{O}_{w}\right\}$ is an affine space over $\mathbf{k}$ of dimension $l(w)$. The subset $S$ of $W$ is then given by $\{s \in W ; l(w)=1\}$. If $B \in \mathcal{B}$, then $F(B) \in \mathcal{B}$; moreover, $B \mapsto F(B)$ is a bijection $F: \mathcal{B} \rightarrow \mathcal{B}$.

For a finite set $X$ we denote by $|X|$ the cardinal of $X$. If $X \subset X^{\prime}$ are sets and $f: X^{\prime} \rightarrow X^{\prime}$ is a map such that $f(X) \subset X$ we set $X^{f}=\{x \in X ; f(x)=x\}$.

2.2. Let $s \in S$. If $B, B^{\prime} \in \mathcal{B}$ we write $B \sim_{s} B^{\prime}$ if $\left(B, B^{\prime}\right) \in \mathcal{O}_{1} \cup \mathcal{O}_{s}$. This is an equivalence relation on $\mathcal{B}$. A subgroup $P$ of $\mathbf{G}$ is said to be parabolic of type $s$ if it is the union of all $B$ in a fixed equivalence class for $\sim_{s}$. Let $\mathcal{P}_{s}$ be the set of parabolic subgroups of type $s$ of $\mathbf{G}$. For $P \in \mathcal{P}_{s}$ we set $\mathcal{B}_{P}=\{B \in \mathcal{B} ; B \subset P\}$; this is a projective line over $\mathbf{k}$.

If $P \in \mathcal{P}_{s}$ and $B \in \mathcal{B}$, then there is a unique element $y=\operatorname{pos}(B, P) \in W$ such that $y<y s$ and $\left(B, B^{\prime}\right) \in \mathcal{O}_{y} \cup \mathcal{O}_{y s}$ for any $B^{\prime} \in \mathcal{B}_{P}$; moreover, we have $\left(B, B^{\prime}\right) \in \mathcal{O}_{y}$ for a unique $B^{\prime} \in \mathcal{B}_{P}$, denoted by $P^{B}$.

2.3. Let $s, s^{\prime} \in S$ and let $P \in \mathcal{P}_{s}, P^{\prime} \in \mathcal{P}_{s^{\prime}}$. There is a unique element $y=$ $\operatorname{pos}\left(P, P^{\prime}\right) \in W$ such that $y<s y, y<y s^{\prime}$ and $\left(B, B^{\prime}\right) \in \mathcal{O}_{y} \cup \mathcal{O}_{s y} \cup \mathcal{O}_{y s^{\prime}} \cup \mathcal{O}_{s y s^{\prime}}$ for any $B \in \mathcal{B}_{P}, B^{\prime} \in \mathcal{B}_{P^{\prime}}$. Assuming in addition that $s y=y s^{\prime}$, the map $\mathcal{B}_{P} \rightarrow \mathcal{B}_{P^{\prime}}$ given by $B \mapsto P^{\prime B}$ is an isomorphism of projective lines with inverse $B^{\prime} \mapsto P^{B^{\prime}}$. 
2.4. We shall fix $C \in \mathcal{B}^{F}$. We assume that $*$ has the following property: we can find an involutive automorphism of algebraic groups $\iota: \mathbf{G} \rightarrow \mathbf{G}$ such that:

$\iota$ commutes with $F: \mathbf{G} \rightarrow \mathbf{G}$;

$\iota(C)=C$;

if $\left(B, B^{\prime}\right) \in \mathcal{O}_{w}$, then $\left(\iota(B), \iota\left(B^{\prime}\right)\right) \in \mathcal{O}_{w^{*}}$.

Note that $\iota$ defines an involution $\mathcal{B} \rightarrow \mathcal{B}$ of algebraic varieties (denoted again by $\iota$ ). It commutes with $F: \mathcal{B} \rightarrow \mathcal{B}$.

2.5. For $(z, y) \in \mathbf{I}_{*} \times W$ we set

$$
\mathcal{X}_{y}^{z}=\left\{B \in \mathcal{B}^{F^{2}} ;(B, F(\iota(B))) \in \mathcal{O}_{z},(C, B) \in \mathcal{O}_{y}\right\} .
$$

This is a finite set.

Proposition 2.6. Let $(z, y) \in \mathbf{I}_{*} \times W, s \in S$. Assume that $s z<z$. We have

(i) $(p+1)\left|\mathcal{X}_{y}^{z}\right|=-p\left|\mathcal{X}_{y}^{s z}\right|+\left|\mathcal{X}_{y s}^{s z}\right|$ if $s z=z s^{*}, y<y s$;

(ii) $(p+1)\left|\mathcal{X}_{y}^{z}\right|=p^{2}\left|\mathcal{X}_{y s}^{s z}\right|+\left(p^{2}-p-1\right)\left|\mathcal{X}_{y}^{s z}\right|$ if $s z=z s^{*}, y>y s$;

(iii) $\left|\mathcal{X}_{y}^{z}\right|=\left|\mathcal{X}_{y s}^{s z s^{*}}\right|$ if $s z \neq z s^{*}, y<y s$;

(iv) $\left|\mathcal{X}_{y}^{z}\right|=p^{2}\left|\mathcal{X}_{y s}^{s z s^{*}}\right|+\left(p^{2}-1\right)\left|\mathcal{X}_{y}^{s z s^{*}}\right|$ if $s z \neq z s^{*}, y>y s$.

In the setup of (iii), (iv) we have $l(z)=l(s)+l\left(s z s^{*}\right)+l\left(s^{*}\right)$; hence for any $B \in \mathcal{X}_{y}^{z}$ we have $\left(B, \beta_{B}\right) \in \mathcal{O}_{s},\left(\beta_{B}, \beta_{B}^{\prime}\right) \in \mathcal{O}_{s z s^{*}},\left(\beta_{B}^{\prime}, F(\iota(B))\right) \in \mathcal{O}_{s^{*}}$ for uniquely defined $\beta_{B}, \beta_{B}^{\prime}$ in $\mathcal{B}$. It follows that $\left(B, F\left(\iota\left(\beta_{B}^{\prime}\right)\right)\right) \in \mathcal{O}_{s},\left(F\left(\iota\left(\beta_{B}^{\prime}\right)\right), F\left(\iota\left(\beta_{B}\right)\right)\right) \in$ $\mathcal{O}_{s z s^{*}},\left(F\left(\iota\left(\beta_{B}\right)\right), B\right) \in \mathcal{O}_{s^{*}}$ and from the uniqueness we see that $\beta_{B}^{\prime}=F\left(\iota\left(\beta_{B}\right)\right)$, $\beta_{B}=F\left(\iota\left(\beta_{B}^{\prime}\right)\right)$; hence $F^{2}\left(\beta_{B}\right)=\beta_{B}$.

Assume that we are in the setup of (iii). We have a bijection $\mathcal{X}_{y}^{z} \rightarrow \mathcal{X}_{y s}^{s z s^{*}}$ given by $B \mapsto \beta_{B}$. Hence (iii) holds.

Assume that we are in the setup of (iv). We have a map $\mathcal{X}_{y}^{z} \rightarrow \mathcal{X}_{y}^{s z s^{*}} \cup \mathcal{X}_{y s}^{s z s^{*}}$ given by $B \mapsto \beta_{B}$. Its fibre over a point in $\mathcal{X}_{y}^{s z s^{*}}$ has cardinal $p^{2}-1$ while its fibre over a point in $\mathcal{X}_{y s}^{s z s^{*}}$ has cardinal $p^{2}$. We deduce that (iv) holds.

In the rest of the proof we assume that $s z=z s^{*}$. We set $y^{\prime}=y$ if $y<y s$ and $y^{\prime}=y s$ if $y s<y$. Let

$$
Y=\left\{P \in \mathcal{P}_{s} ; F^{2}(P)=P, \operatorname{pos}(P, F(\iota(P)))=s z, \operatorname{pos}(C, P)=y^{\prime}\right\} .
$$

For $P \in Y$ we have $P^{C} \in \mathcal{B}_{P},(F(P))^{C}=F\left(P^{C}\right) \in \mathcal{B}_{F(P)}$.

We define a map $\mathcal{X}_{y^{\prime}}^{z} \cup \mathcal{X}_{y^{\prime}}^{s z} \cup \mathcal{X}_{y^{\prime} s}^{z} \cup \mathcal{X}_{y^{\prime} s}^{s z} \rightarrow Y$ by $B \mapsto P$ where $B \subset P \in \mathcal{P}_{s}$. The fibre of this map over $P \in Y$ is $\left\{\beta \in \mathcal{B}^{F^{2}} ; B \subset P\right\}$, hence it has cardinal $p^{2}+1$.

We define a bijection $\mathcal{X}_{y^{\prime}}^{z} \cup \mathcal{X}_{y^{\prime}}^{s z} \rightarrow Y$ by $B \mapsto P$ where $B \subset P \in \mathcal{P}_{s}$.

We define a map $\mathcal{X}_{y^{\prime}}^{s z} \cup \mathcal{X}_{y^{\prime} s}^{s z} \rightarrow Y$ by $B \mapsto P$ where $B \subset P \in \mathcal{P}_{s}$. The fibre of this map over $P \in Y$ is $\left\{\beta \in \mathcal{B}^{F^{2}} ; \beta \subset P,(\beta, F(\beta)) \in \mathcal{O}_{s z}\right\}$ which can be viewed as a set of $\mathbf{F}_{p}$-rational points of the projective line $\mathcal{B}_{P}$ with Frobenius map $\beta \mapsto P^{F(\beta)}$; hence it has cardinal $p+1$.

We see that:

$\left|\mathcal{X}_{y^{\prime}}^{z}\right|+\left|\mathcal{X}_{y^{\prime}}^{s z}\right|+\left|\mathcal{X}_{y^{\prime} s}^{z}\right|+\left|\mathcal{X}_{y^{\prime} s}^{s z}\right|=\left(p^{2}+1\right)|Y|$,

$\left|\mathcal{X}_{y^{\prime}}^{z}\right|+\left|\mathcal{X}_{y^{\prime}}^{s z}\right|=|Y|$,

$\left|\mathcal{X}_{y^{\prime}}^{s z}\right|+\left|\mathcal{X}_{y^{\prime} s}^{s z}\right|=(p+1)|Y|$.

Thus we have:

$$
\begin{aligned}
& \left|\mathcal{X}_{y^{\prime}}^{x}\right|+\left|\mathcal{X}_{y^{\prime}}^{s z}\right|+\left|\mathcal{X}_{y^{\prime} s}^{z}\right|+\left|\mathcal{X}_{y^{\prime} s}^{s z}\right|=\left(p^{2}+1\right)\left(\left|\mathcal{X}_{y^{\prime}}^{z}\right|+\left|\mathcal{X}_{y^{\prime}}^{s z}\right|\right) \\
& \left|\mathcal{X}_{y^{\prime}}^{s z}\right|+\left|\mathcal{X}_{y^{\prime} s}^{s z}\right|=(p+1)\left(\left|\mathcal{X}_{y^{\prime}}^{z}\right|+\left|\mathcal{X}_{y^{\prime}}^{s z}\right|\right)
\end{aligned}
$$

This implies that (i),(ii) hold. 
Proposition 2.7. Let $(z, y) \in \mathbf{I}_{*} \times W, s \in S$. Assume that ys $<y$. We have

(i) $\mathcal{X}_{y}^{z}=p \mathcal{X}_{y s}^{z}+(p+1) \mathcal{X}_{y s}^{s z}$ if $s z=z s^{*}>z$;

(ii) $\mathcal{X}_{y}^{z}=\left(p^{2}-p-1\right) \mathcal{X}_{y s}^{z}+\left(p^{2}-p\right) \mathcal{X}_{y s}^{s z}$ if $s z=z s^{*}<z$;

(iii) $\mathcal{X}_{y}^{z}=\mathcal{X}_{y s}^{s z s^{*}}$ if $s z \neq z s^{*}>z$;

(iv) $\mathcal{X}_{y}^{z}=\left(p^{2}-1\right) \mathcal{X}_{y s}^{z}+p^{2} \mathcal{X}_{y s}^{s z s^{*}}$ if $s z \neq z s^{*}<z$.

The proposition is deduced from Proposition 2.6 in the same way as Proposition 1.4 was deduced from 1.2 (or rather in reverse).

Proposition 2.8. For any $z \in \mathbf{I}_{*}$ we have $\left|\mathcal{X}_{1}^{z}\right|=\delta_{z, 1}$.

We have

$$
\mathcal{X}_{1}^{z}=\left\{B \in \mathcal{B}^{F^{2}} ;(B, F(\iota(B))) \in \mathcal{O}_{z}, C=B\right\} .
$$

Since $C=F(\iota(C))$ we have $(C, F(\iota(C))) \in \mathcal{O}_{1}$; hence $\mathcal{X}_{1}^{z}$ is a single point $C$ if $z=1$ and is empty if $z \neq 1$. The proposition is proved.

Proposition 2.9. For any $y \in W$ we have $\left|\mathcal{X}_{y}^{1}\right|=\delta_{y, y^{*}} p^{l(y)}$.

We have

$$
\begin{aligned}
\mathcal{X}_{y}^{1} & =\left\{B \in \mathcal{B}^{F^{2}} ; B=F(\iota(B)),(C, B) \in \mathcal{O}_{y}\right\} \\
& =\left\{B \in \mathcal{B} ; B=F(\iota(B)),(C, B) \in \mathcal{O}_{y}\right\} .
\end{aligned}
$$

If $B \in \mathcal{X}_{y}^{1}$, then $\left(F(\iota(C)), F(\iota(B)) \in \mathcal{O}_{y^{*}}\right.$, hence $(C, B) \in \mathcal{O}_{y^{*}}$ so that $y=y^{*}$. Thus, if $\mathcal{X}_{y}^{1} \neq \emptyset$, then $y=y^{*}$. Assume now that $y=y^{*}$. Consider the $l(y)$ dimensional affine space $\left\{B \in \mathcal{B} ;(C, B) \in \mathcal{O}_{y}\right\}$ over k. Since $y=y^{*}$, this affine space is stable under $B \mapsto F(\iota(B))$ which is the Frobenius map for an $\mathbf{F}_{p}$-rational structure whose fixed point set is exactly $\mathcal{X}_{y}^{1}$. It follows that $\left|\mathcal{X}_{y}^{1}\right|=p^{l(y)}$. The proposition is proved.

Proposition 2.10. For any $(z, y) \in \mathbf{I}_{*} \times W$ we have $\left|\mathcal{X}_{y}^{z}\right|=\left.X_{y}^{z}\right|_{u=p}$.

By Propositions 1.2 and 2.8, the two sides of the equality in the proposition satisfy the same inductive formulas; they also satisfy the same initial conditions $\left|\mathcal{X}_{1}^{z}\right|=\left.X_{1}^{z}\right|_{u=p}=\delta_{z, 1}$ (see 2.8 and 1.2). Hence they are equal. The proposition is proved.

Proposition 2.11. For any $y \in W$ we have $X_{y}^{1}=\delta_{y, y^{*}} u^{l(y)}$.

Combining Propositions 2.9 and 2.10 we see that $\left.X_{y}^{1}\right|_{u=p}=\left.\delta_{y, y^{*}} u^{l(y)}\right|_{u=p}$. Since two polynomials in $u$ which take equal values at any prime number are equal, we deduce that $X_{y}^{1}=\delta_{y, y^{*}} u^{l(y)}$. The proposition is proved.

Note that this proof gives an alternative approach to Proposition 1.8 in our case.

\section{REFERENCES}

[L1] G. Lusztig, A bar operator for involutions in a Coxeter group, Bull. Inst. Math. Acad. Sin. (N.S.) 7 (2012), no. 3, 355-404. MR3051318

[L2] G. Lusztig, Hecke algebras with unequal parameters, CRM Monograph Series, vol. 18, American Mathematical Society, Providence, RI, 2003. MR1974442 (2004k:20011)

[LV] G. Lusztig and D. Vogan Jr., Hecke algebras and involutions in Weyl groups, Bull. Inst. Math. Acad. Sin. (N.S.) 7 (2012), no. 3, 323-354. MR3051317

[MW] E. Marberg and G. White, Variations of the Poincaré series for the affine Weyl groups and q-analogues of Chebyshev polynomials, arxiv:1410.2772.

Department of Mathematics, M.I.T., Cambridge, Massachusetts 02139

E-mail address: gyuri@math.mit.edu 